\title{
Recurrent Episodes of Nivolumab-Induced Pneumonitis after Nivolumab Discontinuation and the Time Course of Carcinoembryonic Antigen Levels: A Case of a 58-Year-Old Woman with Non-Small Cell Lung Cancer
}

\author{
Corine de Jong $^{\mathrm{a}} \quad$ Bas J.M. Peters ${ }^{\mathrm{a}} \quad$ Franz M.N.H. Schramel ${ }^{\mathrm{b}}$ \\ ${ }^{a}$ Department of Clinical Pharmacology, St. Antonius Hospital, Nieuwegein, The Netherlands; ${ }^{b}$ Department of \\ Pulmonology, St. Antonius Hospital, Nieuwegein, The Netherlands
}

\section{Established Facts}

- Immune checkpoint inhibitors can cause serious adverse events that are mostly autoimmune related, like pneumonitis.

- Recurrent episodes of pneumonitis can even occur after discontinuation of immunotherapy.

\section{Novel Insights}

- Carcinoembryonic antigen (CEA) serum levels seem to reflect antitumour response of immunotherapy even after immunotherapy discontinuation.

- The clinical utility of CEA serum levels, combined with other biomarkers, for identifying subgroups of patients differing in survival, response rate, and development of immune-related adverse events should be the topic of further research to extend personalized medicine.

\section{Keywords}

Nivolumab · Pneumonitis · Checkpoint inhibitor · Immunerelated adverse event $\cdot$ Carcinoembryonic antigen

\section{Abstract \\ Introduction: The introduction of immune checkpoint in- hibitors heralded a new era in the treatment of non-small cell lung cancer. However, nivolumab, an anti-PD-1 antibody, can cause serious adverse events that are mostly autoim- mune related. Case Presentation: A 58-year-old woman was}

treated with nivolumab as second-line therapy for stage IV adenocarcinoma. The patient developed a nivolumab-induced recurrent pneumonitis preceding durable clinical remission after seven cycles of nivolumab. Although highdose glucocorticosteroids were tapered to conform to contemporary guidelines, recurring episodes of pneumonitis occurred without nivolumab rechallenge. In addition, carcinoembryonic antigen (CEA) serum levels were associated with treatment response, since CEA decline correlated with a near complete radiological response and, conversely, elevated CEA serum levels were associated with progressive

\section{KARGER}

(c) 2018 S. Karger AG, Basel

E-Mail karger@karger.com

www.karger.com/che
Franz M.N.H. Schramel, MD, PhD

Department of Pulmonology, St. Antonius Hospital Koekoekslaan 1

NL-3435 CM Nieuwegein (The Netherlands)

E-Mail f.schramel@ antoniusziekenhuis.nl 
disease. Conclusions: In this case, we described recurrence of nivolumab-induced pneumonitis as a serious adverse event in immune checkpoint inhibitors. Our case illustrates that immune-related adverse events may correlate with antitumour activity, even after treatment discontinuation. In addition, this case suggests the possible clinical utility of CEA serum levels for the assessment of (durable) effects of immunotherapy.

(c) 2018 S. Karger AG, Basel

\section{Introduction}

The introduction of immune checkpoint inhibitors heralded a new era in the treatment of non-small cell lung cancer (NSCLC). Nivolumab, a human immunoglobulin G4 anti-programmed death (PD)-1 monoclonal antibody, can provide long-term clinical benefit with significant better overall survival and progression-free survival than docetaxel in the second-line treatment of both squamous and non-squamous NSCLC, although reliable biomarkers for predicting the effect of nivolumab are urgently required [1]. In general, nivolumab is better tolerated than conventional chemotherapy, accompanied by a manageable adverse event profile. These adverse events are mostly autoimmune related, including endocrinopathies, pruritis, and vitiligo [2]. More severe events like colitis, nephritis, hepatitis, and pneumonitis have also been reported [2]. Pneumonitis (CTC any grade) occurred in 5\% of NSCLC patients in the pivotal trial; however, the aetiology has not been fully elucidated [3]. Although immunotherapy-induced toxicity is generally reversible after glucocorticosteroid treatment [2], we describe a patient with recurrent episodes of pneumonitis after discontinuation of nivolumab and initial successful treatment with glucocorticosteroid without nivolumab rechallenge, including a follow-up of carcinoembryonic antigen (CEA) levels, a glycoprotein involved in the modulation of cellular processes, cell-cell recognition, cell adhesion, and malignancy [4].

\section{Case Report}

\section{History and Initial Presentation}

In November 2011, a 58-year-old Caucasian woman (former smoker, 40 pack-years, no medical history, no intake of any pneumotoxic agents) was treated with lobectomy and adjuvant chemotherapy because of a stage IIB squamous cell carcinoma of the upper lobe. Follow-up was uneventful until June 2015. The scheduled computed tomography (CT) scan of June 2015 was suspected for relapse. Positron emission tomography (PET)-CT and further diagnostics confirmed a second primary malignancy: stage IV ade- nocarcinoma (cT3N3M1a). Next generation sequencing of the tissue revealed no KRAS, epidermal growth factor receptor (EGFR), BRAF, ERBB2 mutations, or anaplastic lymphoma kinase (ALK) rearrangement. Chemotherapy was initiated consisting of carboplatin, paclitaxel, and bevacizumab. After four cycles, partial response of the primary tumour was evident by radiological evaluation. Treatment was continued with bevacizumab maintenance therapy. PET-CT follow-up showed only fluorodeoxyglucose (FDG) uptake in the primary tumour and one localization of a right-sided pleural metastasis which were treated with stereotactic radiotherapy (60 Gy). In June 2016, an elevated CEA serum level was detected $(149 \mu \mathrm{g} / \mathrm{L})$. Follow-up PET-CT in July 2016 showed tumour progression which was confirmed by VATS with excision of lymph nodes (2 and 4$)$ and pericardial tissue which revealed metastases of adenocarcinoma.

\section{Immunotherapy and Pneumonitis}

In September 2016, nivolumab $3 \mathrm{mg} / \mathrm{kg}$ every 2 weeks was initiated inducing a prompt and near complete response, as confirmed on the CT scan of December 2016. Radiological response was associated with tumour marker decline (CEA serum level prior to nivolumab [September 2016] and CEA serum level after four cycles of nivolumab [November 2016] of 385 and $180 \mu \mathrm{g} / \mathrm{L}$, respectively). After the seventh cycle of nivolumab in December 2016, the patient presented with malaise, joint pain, fever, nausea, and reduced intake. The patient had no pre-existing interstitial pneumonia, a potential risk factor for drug-induced pneumonitis. Radiological evaluation in January 2017 showed pulmonary infiltrates, suspected for pneumonia or immune-related pneumonitis (CTC grade 3) as shown in Figure 1. At that point, CEA levels were further declined to $42 \mu \mathrm{g} / \mathrm{L}$.

Subsequently, nivolumab was discontinued and empiric antimicrobial therapy and high-dose oral prednisone $(50 \mathrm{mg} /$ day $)$ was started during admission. Because of clinical improvement, prednisone was reduced by $10 \mathrm{mg} /$ day weekly and the patient was discharged. In February 2017, just after prednisone discontinuation, she was hospitalized again for grade 3 pneumonitis and prednisone treatment was restarted. Notable, during the following months, recurrent episodes of pneumonitis at low-dose glucocorticoids occurred for which hospitalization was indicated (Fig. 2). These manifestations developed without nivolumab re-administration, a potential trigger for relapse [2]. A CT scan at the time of a recurrent episode of pneumonitis (May 2017) showed even more peribronchovascular consolidations and ground-glass opacities, which confirmed the immune-mediated adverse event (Fig. 1).

Besides ongoing immune-related toxicity, the patient achieved a near complete response, while immunohistochemistry staining of the pericardial tissue of the poorly differentiated adenocarcinoma revealed no PD-L1 expression (PD-L1 <1\%). Durable tumour response to a near complete remission was confirmed by radiographic assessment, without evidence of recurrence or metastasis until 11 months after discontinuation of nivolumab and despite the use of high-dose glucocorticosteroids. Unfortunately, in November 2017, a CT scan of the thorax and abdomen was performed and showed progressive disease with symptomatic multiple metastasis. Elevated CEA serum levels were detected since May 2017 as shown in Figure 2. One and a half years after the initial diagnosis of NSCLC, palliative radiotherapy was initiated and subsequently, in January 2018, treatment was continued with pemetrexed once every 3 weeks, while CEA serum level was increased to $561 \mu \mathrm{g} / \mathrm{L}$. 

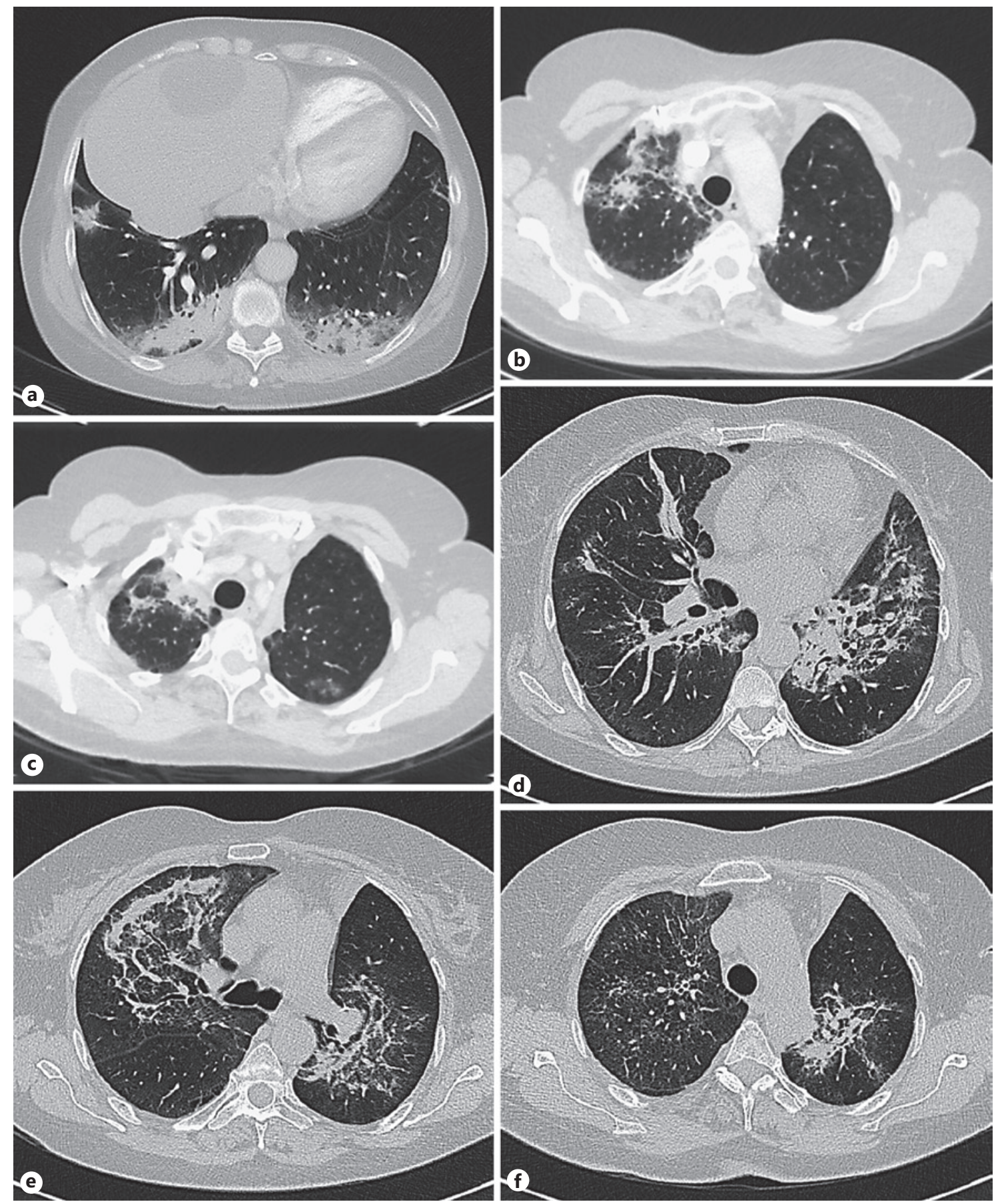

Fig. 1. Chest computed tomography (CT) scan after seven cycles of nivolumab (January 2017). The restaging CT scan revealed lung abnormalities typical for drug-induced symptomatic pneumonitis (CTC grade 3), with multiple consolidations and ground-glass opacities in right upper and right lower lobe and left lower lobe, in which traction bronchiectasis is seen at several levels $(\mathbf{a}-\mathbf{c})$. This pattern reflects the radiologic pattern of an organizing pneumonia, which is often the presentation in nivolumab-induced pneumonitis. Radiological evaluation at the time of a subsequent episode of pneumonitis (May 2017) showed even more peribronchovascular consolidations and ground-glass opacities (d-f) which confirmed the immune-mediated adverse event. 


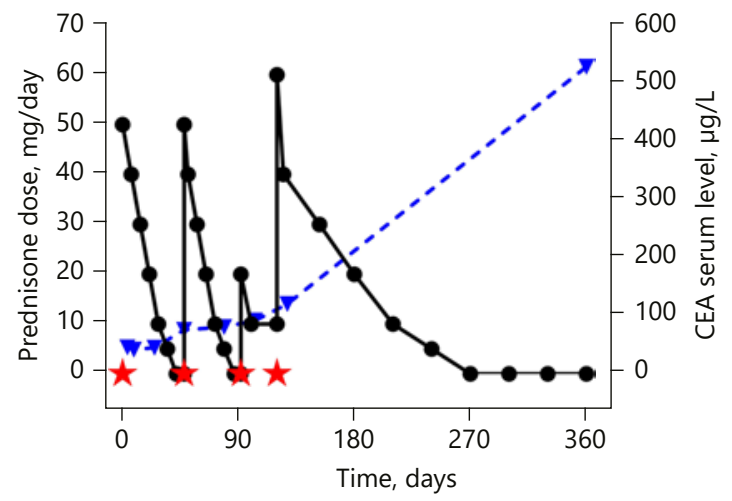

Fig. 2. Recurrence of nivolumab-induced pneumonitis and oral steroid treatment. Time was measured in days after discontinuation of nivolumab and initiation of oral high-dose prednisone for pneumonitis (CTC grade 3) in January 2017 (day 0). Stars indicate exacerbation of pneumonitis, primarily accompanied by malaise, joint paint, fever and nausea. From May 2017 (day 120), glucocorticoid dosage was adjusted to high-dose prednisone $(60 \mathrm{mg} /$ day $)$ for 1week, followed by a reduction of $10 \mathrm{mg} /$ day every month. Since glucocorticoids were reduced more gradually, no recurrence of pneumonitis occurred. The blue line indicates carcinoembryonic antigen (CEA) serum levels $(\mu \mathrm{g} / \mathrm{L})$.

\section{Discussion/Conclusion}

Here we describe a patient with recurrent episodes of pneumonitis after discontinuation of nivolumab, including a time course of CEA levels. In our case, severe pneumonitis (CTC grade 3 ) occurred approximately 3 months after initiation of nivolumab. Generally, immune-related adverse events (irAE) develop within the first few weeks to months after treatment initiation [5]. Previous research by Naidoo et al. [6], including 915 patients who received anti-PD-1/PD-L1 monotherapy or in combination with anti-cytotoxic T-cell lymphocyte- $4 \mathrm{mAb}$, has shown a median time of onset of pneumonitis of 2.8 months (range 9 days to 19.2 months), which is in line with the time of onset of pneumonitis in our case. However, our case report points out that clinicians should be aware of durable irAE, even after discontinuation of immune checkpoint inhibitors.

Recently, Brahmer et al. [7] conducted a systematic review to outline strategies and offer guidance on the recommended management of irAE in patients treated with immune checkpoint inhibitors. Recommendations of this systematic review included initiation of high-dose glucocorticosteroids (prednisone $1 \mathrm{mg} / \mathrm{kg}$ /day per os) in case of grade 3 toxicities, where glucocorticosteroids should be tapered (5-10 mg/week) over the course of at least 4-6 weeks. Refractory cases may require infliximab or other immunosuppressive therapy. Since in our case glucocorticoids were, initially and at resumption, effective as first-line immunosuppressive agent, no additional immunosuppressive agent was added to the treatment. Nevertheless, our case illustrates that although high-dose glucocorticoids were reduced to conform to guidelines, recurrent pneumonitis may occur. The observed recurrent episodes of pneumonitis may have been caused by tapering glucocorticoid treatment $10 \mathrm{mg} /$ week, whereas $5 \mathrm{mg} /$ week may have been more appropriate after the first recurrent episode of pneumonitis. Therefore, it can be advocated that in patients with recurrent pneumonitis steroids need to be reduced more gradually.

Currently, no data are available to select patients at high risk for (early or late onset) irAE in clinical practice. For instance, HLA-A status has not been related to the likelihood of irAE among patients treated with immune checkpoint inhibitors [5]. Microbiologic composition of a patient's gastrointestinal flora, however, has been investigated in preclinical and clinical settings, suggesting it to be associated with treatment efficacy and the likelihood to development irAE [5]. Indeed, Dubin et al. [8] found increased representation of bacteria belonging to the Bacteroidetes phylum to be correlated with a lower incidence of checkpoint-blockade-induced colitis in 34 patients with metastatic melanoma undergoing ipilimumab treatment. In addition, concomitant use of antibiotics (affecting the composition of the gut microbiota) during initiation of immune checkpoint inhibitors has been evaluated in 30 NSCLC patients with respect to clinical outcomes and irAE [9]. Although not statistically significant, irAE occurred in 27.3 and $57.9 \%$ in patients with and without antibiotic use, respectively [9]. Conversely, antibiotics use has also been associated with reduced efficacy $[9,10]$. Additional research is needed to determine whether influencing of the gastrointestinal flora, for example through dietary intervention or the use of probiotics or antibiotics, could reduce the risk of irAE without affecting the response to checkpoint inhibitors [5].

In addition, present biomarkers for predicting response to checkpoint inhibitors, including PD-L1 expression and mutational burden, show incomplete predictive performance [11]. Nevertheless, promising research is focused on comprehensive immune profiling. As described by Morrison et al. [12], ascertaining the effects of checkpoint inhibitors requires a complex and multifactorial approach, since the wide range of immunosuppressive and 
activating mechanisms are not yet fully understood. Probably, as one of these multifactorial aspects, serum biomarkers can be of added value in the prediction of ( $\mathrm{du}$ rable) effects of immunotherapy. Recently, pre-treatment CEA serum levels were found to be biomarkers associated with benefit of nivolumab on the basis of a retrospective cohort trial consisting of 189 patients with NSCLC conducted by Kataoka et al. [13]. Routine determination of CEA levels during treatment follow-up is part of our hospital's standard of care, enabling us to describe the time course of CEA levels in this case. CEA levels were associated with treatment response, since CEA decline correlated with a near complete radiological response and, conversely, elevated CEA serum levels were associated with progressive disease. Remarkable, as shown in Figure 2, elevated CEA levels seem to correlate with progressive disease and simultaneous resolution of the recurrent pneumonitis, suggesting an earlier described correlation between both durable response and durable toxicity [14]. Therefore, early in-treatment as well as CEA measurements during follow-up may be helpful in considering the optimal treatment duration of immune checkpoint inhibitors. Although it is still unclear whether re-administration after severe toxicities (CTC grade 3 ) should be recommended [12], CEA serum levels possibly reflect antitumour responses and can encourage clinicians in the process of evaluating (durable) treatment effects in anticipation of radiological evaluation. The clinical utility of CEA serum levels, combined with other biomarkers, for identifying subgroups of patients differing in survival, response rate, and development of irAE should be the topic of further research.

In conclusion, recurrence of nivolumab-induced pneumonitis can occur as a serious adverse event in immune checkpoint inhibitors. Our case illustrates that
irAE may correlate with antitumour activity, even long after treatment discontinuation. Besides, this case suggests the possible clinical utility of CEA serum levels for the assessment of (durable) effects of immunotherapy. Hence, risk stratification and aetiology of immune-related toxicities and biomarkers for response to immunotherapy are important topics for future research to extend personalized medicine.

\section{Acknowledgements}

The authors would like to thank F.T. van Beek, MD, Interstitial Lung Diseases Center of Excellence, Department of Pulmonology, St. Antonius Hospital, Nieuwegein, The Netherlands, for additional radiographic assessment.

\section{Statement of Ethics}

The authors have no ethical conflicts to disclose.

\section{Disclosure Statement}

The authors have no conflicts of interest to declare.

\section{Funding Sources}

The authors have nothing to declare.

\section{Author Contributions}

C.J. made the first draft of the manuscript. B.P. and F.S. critically reviewed and revised the article. The final version of the paper was seen and approved by all authors.

\section{References}

1 Horn L, Spigel DR, Vokes EE, Holgado E, Ready N, Steins M, et al. Nivolumab Versus Docetaxel in Previously Treated Patients With Advanced Non-Small-Cell Lung Cancer: Two-Year Outcomes From Two Randomized, Open-Label, Phase III Trials (CheckMate 017 and CheckMate 057). J Clin Oncol. 2017 Dec;35(35):3924-33.

2 Bristol-Myers Squibb Company. ImmuneMediated Adverse Reactions Management Guide 2017.

3 Brahmer J, Reckamp KL, Baas P, Crinò L, Eberhardt WE, Poddubskaya E, et al. Nivolumab versus Docetaxel in Advanced Squamous-
Cell Non-Small-Cell Lung Cancer. N Engl J Med. 2015 Jul;373(2):123-35.

4 Grunnet M, Sorensen JB. Carcinoembryonic antigen (CEA) as tumor marker in lung cancer. Lung Cancer. 2012 May;76(2):138-43.

5 Postow MA, Sidlow R, Hellmann MD. Immune-Related Adverse Events Associated with Immune Checkpoint Blockade. N Engl J Med. 2018 Jan;378(2):158-68.

6 Naidoo J, Wang X, Woo KM, Iyriboz T, Halpenny D, Cunningham J, et al. Pneumonitis in Patients Treated With Anti-Programmed Death-1/Programmed Death Ligand 1 Therapy. J Clin Oncol. 2017 Mar;35(7):709-17.
7 Brahmer JR, Lacchetti C, Schneider BJ, Atkins MB, Brassil KJ, Caterino JM, et al.; National Comprehensive Cancer Network. Management of Immune-Related Adverse Events in Patients Treated With Immune Checkpoint Inhibitor Therapy: American Society of Clinical Oncology Clinical Practice Guideline. J Clin Oncol. 2018 Jun;36(17): 1714-68.

8 Dubin K, Callahan MK, Ren B, Khanin R, Viale A, Ling L, et al. Intestinal microbiome analyses identify melanoma patients at risk for checkpoint-blockade-induced colitis. Nat Commun. 2016 Feb;7:10391. 
9 Huemer F, Rinnerthaler G, Westphal T, Hackl H, Hutarew G, Gampenrieder SP, et al. Impact of antibiotic treatment on immunecheckpoint blockade efficacy in advanced non-squamous non-small cell lung cancer. Oncotarget. 2018 Mar;9(23):16512-20.

10 Routy B, Le Chatelier E, Derosa L, Duong CP, Alou MT, Daillère R, et al. Gut microbiome influences efficacy of PD-1-based immunotherapy against epithelial tumors. Science. 2018 Jan;359(6371):91-7.
11 Hellmann MD, Ciuleanu TE, Pluzanski A, Lee JS, Otterson GA, Audigier-Valette C, et al. Nivolumab plus Ipilimumab in Lung Cancer with a High Tumor Mutational Burden. N Engl J Med. 2018 May;378(22):2093-104.

12 Morrison C, Pabla S, Conroy JM, Nesline MK, Glenn ST, Dressman D, et al. Predicting response to checkpoint inhibitors in melanoma beyond PD-L1 and mutational burden. J Immunother Cancer. 2018 May;6(1):32.
13 Kataoka Y, Hirano K, Narabayashi T, Hara S, Fujimoto D, Tanaka T, et al. Carcinoembryonic Antigen as a Predictive Biomarker of Response to Nivolumab in Non-small Cell Lung Cancer. Anticancer Res. 2018 Jan;38(1):55963.

14 Haratani K, Hayashi H, Chiba Y, Kudo K, Yonesaka K, Kato R, et al. Association of Immune-Related Adverse Events With Nivolumab Efficacy in Non-Small-Cell Lung Cancer. JAMA Oncol. 2018 Mar;4(3):374-8. 